\title{
Effects of Oral Administration of D-002 (Beeswax Alcohols) on Histological and Functional Outcomes in a Rat Model of Antigen-Induced Arthritis: Preliminary Study
}

\author{
Miriam Noa Puig ${ }^{1 a^{*}}$, Sarahí Mendoza Castaño ${ }^{2 b}$, Rosa Mas Ferreiro ${ }^{3 c}$, \\ Maikel Valle Clara $^{4 \mathrm{~d}}$, Nilda Mendoza Hernansez ${ }^{5 \mathrm{e}}$ \\ ${ }^{1}$ Center of National Products, 198 street e/ 19 and 21 street, Havana, Cuba. \\ amiriam.noa@cnic.edu.cu, b sarahi.mendoza@cnic.edu.cu, crosa.mas@cnic.edu.cu, \\ dmaikel.valle@cnic.edu.cu
}

Keywords: beeswax alcohol, histological changes, antigen-induced arthritis, rats

\begin{abstract}
D-002, a mixture of higher aliphatic beeswax alcohols, has been shown to display antiinflammatory effects associated with the dual inhibition of ciclooxygenase and 5-lipoxygenase. Oral D-002 supplementation has been effective in experimental osteoarthritis, ameliorating all features of joint histopathology. Clinical studies have demonstrated that D-002 reduces osteoarthritis symptoms. However, D-002 effects on experimental models of rheumatoid arthritis (RA) have not been evaluated. To investigate whether D-002 improves histopathological and functional outcomes in a rat model of antigen-induced arthritis. First experiment. Rats were randomized into a negative vehicle-control (sham) and four groups injected with complete Freund's adjuvant (CFA): a positive vehicle-control, three treated with D-002 (50, 200 and $400 \mathrm{mg} / \mathrm{kg} /$ day) for 21 days. Second experiment. Rats were randomized into a sham and four CFA-injected groups: a positive vehiclecontrol, two treated with D-002 (25 and $100 \mathrm{mg} / \mathrm{kg} / \mathrm{day})$, one with methorexate (MTX) $(0.3 \mathrm{mg} / \mathrm{kg})$ for 28 days. Arthritis severity was evaluated by bodyweight loss, decreased exploratory activity and histological changes of tarsal joint and spleen samples in both experiments, except the exploratory activity, assessed only in the first one. CFA injection decreased the bodyweight and the exploratory activity, and induced infiltration of mononuclear cells, pannus formation and vascularity in the tarsal joint of positive control rats. These changes were significantly and markedly ameliorated by D-002 as compared to the positive control. MTX also reversed CFA-induced changes. The reduction of the infiltration of mononuclear cell with D-002 $400 \mathrm{mg} / \mathrm{kg}$ was greater $(80.9 \%)$ than with MTX (66.8\%), but effects on other variables were similar. No abnormalities in spleen samples of D-002-treated groups were detected. This is the first report demonstrating the efficacy of oral treatment with D-002 in a rat model of antigen-induced arthritis. Results suggest that D-002 could help manage RA, but confirmation of such potential benefit requires extensive further research.
\end{abstract}

Abreviation list: RA, Rheumatoid arthritis; CFA, complete Freund's adjuvant; MTX, methotrexate; COX, cyclooxygenase; NSAID, Non-steroidal anti-inflammatory drugs; 5-LOX, 5lipoxygenase.

\section{Introduction}

Rheumatoid arthritis (RA), a common inflammatory joint disease that affects approximately $1 \%$ of adults worldwide, is a chronic systemic autoimmune inflammatory disease characterized by inflammation of the synovial membrane and progressive destruction of the joint and bone, which is associated to relevant co-morbidities. [1,2] The disease can exhibit an aggressive course, with repeated bouts of pain and stiffness as relevant contributors to the deterioration of the quality of life of the patients, so that after 10 years on disease, more than half of patients are impended to continue their work, and life expectancy is lowered by about 10 years. [3 -5]

The management of RA has been improved in the last decade, but despite such advances, RA remains a progressive and destructive disease that strongly affect the quality of life of the sufferers, with only $20 \%$ of them reaching remission. [4, 5, 6-11] Steroids are used to reduce pain and 
inflammation in RA, while methotrexate (MTX) prevents the development of destructive changes in bones, joints, and organ tissues. [7] Nevertheless, steroids may lead to relatively troublesome adverse side effects, which is particularly true for RA patients under longer term and higher dose therapies. [8, 9] In turn, MTX, a disease-modifying anti-rheumatic drug, is the drug of choice for managing RA in terms of its risk/benefit ratio, safety profile, and costs. [8, 10]

Non-steroidal anti-inflammatory drugs (NSAIDs) (non-selective NSAIDs and COX-2 inhibitors) have been widely used for symptomatic relief of pain and inflammation due to their ability to inhibit cyclooxygenase (COX) enzyme and then prostaglandins (PGs) synthesis, but many of the adverse effects (gastrointestinal, renal) of non-selective NSAIDs are also related to inhibition of PGs production, while COX2-inhibitors increase the cardiovascular risk. [11] Indeed, NSAIDsrelated adverse effects depend of their pharmacologic characteristics, like bioavailability, half-life, and their potency for inhibiting COX-1 and COX-2. [12]

This background supports that search for new effective and safer treatments for managing RA is updated. The first step on this way begins with the assessment of the candidates in experimental models. In such regard, animal models have been used extensively for assessing the potential benefits of treatments on RA. Despite all have inherent limitations, several rodent models have significantly contributed to the current knowledge of the main mechanisms involved in RA and to major advances in its treatment. In generally, these models include models of induced arthritis and models of genetically manipulated or spontaneous arthritis. Models of antigen- (collagen, collagenantibody, zymosan, methylated BSA) -induced arthritis belong to the first group, which includes rat model of complete Freund's adjuvant (CFA)-induced arthritis. [13 - 15]

Experimental studies have shown that D-002 (research code), a mixture of high molecular weight beeswax alcohols, displays anti-inflammatory effects in vivo [16 - 19] that have been associated with the dual inhibition of COX and 5-lipoxygenase (5-LOX). [20, 21] Oral supplementation of D-002 has been effective in experimental osteoarthritis, ameliorating cartilage damage, pannus formation and joint inflammation; [22, 23] and clinical studies have demonstrated that D-002 reduces joint pain, stiffness and improves daily activity in subjects with osteoarthritis symptoms. [24, 25]

The effects of D-002 on experimental models of RA, however, have not been evaluated.

Our aim in this study was to investigate whether D-002 reduces histological features and improves functional outcomes in a rat model of CFA-induced arthritis.

\section{Methods}

\section{Animals}

Male Sprague Dawley rats $(275-350 \mathrm{~g})$ acquired in the National Centre for Laboratory Animals Production (CENPALAB, Havana, Cuba) were adapted for 7 days to the experimental conditions: temperature $20-25^{\circ} \mathrm{C}$, humidity $60 \pm 10 \%$ and light/ dark cycles of $12 \mathrm{~h}$. Food (standard chow pellets from CENPALAB) and water were given ad libitum.

This study was designed and conducted according to the Cuban Guidelines of Animals Handling and the Cuban Code of Good Laboratory Practices (GLP), which follow international guidelines for the use and care of laboratory animals. The study protocol and animal use were approved prior to the study by the Institutional Animal Ethics Committee.

\section{Chemicals and test substance}

The batch of D-002, supplied by the Plants of Natural Products (Havana, Cuba), had the following composition: tetracosanol (7.0\%), hexacosanol $(11.5 \%)$, octacosanol (12.1\%), triacontanol (34.8\%), dotriacontanol (22.5\%) and tetratiacontanol $(2.6 \%)$. Purity (total content of these alcohols) was $90.0 \%$. For dosing, D-002 was suspended in a $\%$ Tween-20/water vehicle.

MTX (Calbiochem, Darmstadt, Germany) was diluted in saline for the experiment.

The complete FA (CFA) (batch 068K8761) (Sigma-Aldrich, Inc. Saint Louis, Missouri, USA) used for inducing the arthritis had $1 \mathrm{~mL}$ of $\mathrm{mg}$ of death and heat-dried Mycobacterium tuberculosum, $0.85 \mathrm{~mL}$ of mineral oil, and $0.15 \mathrm{~mL}$ of mannide monoleate. 


\section{Treatment and experimental design}

Two experiments were performed. First experiment. This experiment explored the effects of different doses of D-002 administered for just 21 days on functional and histopahtological outcomes. Rats were randomized into a negative vehicle-control and four groups injected with CFA: a positive vehicle-control, three treated with D-002 (50, 200 and $400 \mathrm{mg} / \mathrm{kg} / \mathrm{day})$. Second experiment. This experiment was focused in exploring the effects of lower doses of D-002 administered for a longer period (28 days) on the primary efficacy variable, taking MTX as reference treatment. Rats were randomized into a negative vehicle-control and four groups injected with CFA: a positive vehicle-control, two treated with D-002 (25 and $100 \mathrm{mg} / \mathrm{kg} / \mathrm{day})$, one with MTX $0.3 \mathrm{mg} / \mathrm{kg}$ for 28 days. In both experiments treatments commenced at 10 days after disease induction.

Rats were sacrificed at the last day of treatment. Hindpaws and spleen samples were collected for histological evaluation in both experiments, while exploratory activity was also assessed in the first one. Change on joint histopathological features was the primary efficacy variable, while effects on exploratory activity (functional outcome) and spleen histopathology were secondary outcomes.

\section{Arthritis induction}

CFA-induced arthritis was induced by a single injection of $100 \mu \mathrm{L}$ of CFA subcutaneously (sc) at the base of the rat tail. Except MTX, which was given twice weekly only, [26, 27] the treatments (vehicle, D-002) were given daily $(9-10 \mathrm{am})$ by gastric gavage $(1 \mathrm{~mL} / 100 \mathrm{~g}$ bodyweight) for 21 and 28 days in first and second experiment, respectively, commencing at day 10 after CFA injection. [28] At treatment completion, rats were sacrificed under thiopental anesthesia.

\section{Effect on functional outcomes}

The potential analgesic effect of D-002 on paw pain was indirectly evaluated through the animal open field exploratory activity, [29] which was assessed in accordance with Carbajal et al. [30] The rats were placed in a transparent plastic round chamber $(15 \mathrm{~cm}$ height $\mathrm{x} 30 \mathrm{~cm}$ diameter $)$ with a central circle $(10 \mathrm{~cm}$ diameter $)$. The numbers of total vertical rears $(\mathrm{R})$ and the numbers of crosses $(\mathrm{C})$ through the central circle in $6 \mathrm{~min}$ were recorded. The numbers of total vertical $\mathrm{R}$ were defined as the number of times the animal stands on both hind limbs while supporting their entire body weight $[29,30]$. The exploratory activity was estimated as the total of rears and crosses $(\mathrm{R}+\mathrm{C})$.

\section{Histopathological study}

After euthanasia on last day of treatment, the hindpaws were amputated just above the knee and fixed in normal buffered formalin for 24 hours and processed. In brief, samples were decalcified in $0.5 \mathrm{~mol} / \mathrm{L}$ disodium EDTA ( $\mathrm{pH}$ 7.4) dissolution at $4{ }^{\circ} \mathrm{C}$ for 4 weeks, embedded in paraffin, and sectioned in a mid-sagittal plane. The sections of articulation of the tarsal joints were stained with hematoxylin $(\mathrm{H})$ and eosin $(\mathrm{E})$, and were examined microscopically. [31] We also performed the $\mathrm{H}$ and $\mathrm{E}$ staining of spleen specimens.

Two blinded observers evaluated mononuclear cell infiltration, cartilage and bone destruction by pannus formation, and vascularity in synovial tissues in each preparation. For this aim, the following scoring system was used: mononuclear cell infiltration ( 0 no infiltration; 1 mild infiltration; 2 moderate infiltration; 3 severe infiltration); cartilage and bone destruction by pannus formation ( 0 no change; 1 mild change -pannus invasion into cartilage-; 2 moderate change -pannus invasion into cartilage/subchondral bone-; 3 severe change -pannus invasion into the subchondral bone-); and vascularity ( 0 almost no blood vessels; 1 a few blood vessels; 2 some blood vessels; 3 many blood vessels). [32]

Occurrence of inflammatory cells/lesions, lymphoid atrophy and extramedullar hematopoyesis were assessed in spleen samples.

\section{Statistical Analysis}

Comparisons among groups were conducted with the Kruskal Wallis test, and paired comparisons between groups with the Mann Whitney U test. Statistical significance was chosen at $\alpha=0.05$. Data were analyzed with the Statistic software package for Windows (Release 6.1, StatSoft Inc, Tulsa, OK, USA). 


\section{Results}

\section{First experiment}

The injection of CFA into the rat tail decreased significantly the bodyweight of the positive controls as compared to negative control group, but such decrease was significantly attenuated by D-002 (Table 1).

Table 1. First experiment: effects of treatments on rat bodyweights

\begin{tabular}{|l|c|c|c|}
\hline Treatment groups & Baseline & 10 days & 21 days \\
\hline Sham (vehicle) & $326.63 \pm 17.12+$ & $359.63 \pm 17.54+$ & $425.00 \pm 18.28+$ \\
\hline Positive control (vehicle + CFA) & $327.38 \pm 17.90$ & $317.88 \pm 17.90$ & $307.88 \pm 17.90$ \\
\hline D002 50 mg/kg + CFA & $327.88 \pm 17.62+$ & $360.88 \pm 17.62+$ & $415.88 \pm 17.62+$ \\
\hline D002 200 mg/kg + CFA & $324.25 \pm 20.87+$ & $357.25 \pm 20.87+$ & $412.25 \pm 20.87+$ \\
\hline D002 400mg/kg + CFA & $324.13 \pm 24.33+$ & $357.13 \pm 24.33+$ & $418.75 \pm 19.98+$ \\
\hline \\
\multicolumn{2}{|c|}{ CFA Complete Freund adjuvant. Values expressed as mean \pm SD } \\
Treatments were given for 21 day starting at day 10 after CFA injection,
\end{tabular}

CFA injection also decreased significantly the exploratory activity in positive control rats versus the negative control group. CFA injection lowered significantly the numbers of rears, the number of crosses and the total exploratory activity by about $72.5 \%, 52.9 \%$ (crosses) and 59\% (total activity), versus the values of the negative control. In contrast, D-002 administration (200 and 400 $\mathrm{mg} / \mathrm{kg} /$ day) significantly attenuated the CFA-induced exploratory activity impairment (Table 2).

Table 2. First experiment: Effects on the open field exploratory activity

\begin{tabular}{|c|c|c|}
\hline Treatment groups & Baseline & 21 days \\
\hline \multicolumn{3}{|c|}{ Rears } \\
\hline Sham (vehicle) & $16.9 \pm 2.7$ & $21.4 \pm 3.5+++$ \\
\hline Positive control (vehicle + CFA) & $18.5 \pm 2.0$ & $5.9 \pm 1.5$ \\
\hline D002 50mg/kg + CFA & $14.7 \pm 3.3$ & $10.5 \pm 2.1+$ \\
\hline D002 200mg/kg + CFA & $18.4 \pm 3.6$ & $14.3 \pm 2.7++$ \\
\hline D002 400mg/kg + CFA & $17.0 \pm 2.7$ & $14.1 \pm 1.8++$ \\
\hline \multicolumn{3}{|c|}{ Crosses } \\
\hline Sham (vehicle) & $49.2 \pm 9.3$ & $46.9 \pm 4.2+++$ \\
\hline Positive control (vehicle + CFA) & $51.9 \pm 3.5$ & $22.1 \pm 3.0$ \\
\hline D002 50mg/kg + CFA & $38.4 \pm 5.5$ & $29.0 \pm 3.7$ \\
\hline D002 200mg/kg + CFA & $43.1 \pm 8.8$ & $35.4 \pm 5.7+$ \\
\hline $\mathrm{D} 002400 \mathrm{mg} / \mathrm{kg}+\mathrm{CFA}$ & $47.4 \pm 8.4$ & $42.6 \pm 4.4++$ \\
\hline \multicolumn{3}{|l|}{ Rears + Crosses } \\
\hline Sham (vehicle) & $66.1 \pm 11.7$ & $68.3 \pm 7.2+++$ \\
\hline Positive control (vehicle + CFA) & $70.4 \pm 4.03$ & $28.0 \pm 4.0$ \\
\hline D002 50mg/kg + CFA & $53.10 \pm 8.6$ & $39.5 \pm 5.2$ \\
\hline D002 200mg/kg + CFA & $61.5 \pm 12.1$ & $49.7 \pm 8.2+$ \\
\hline $\mathrm{D} 002$ 400mg/kg + CFA & $64.4 \pm 10.4$ & $56.7 \pm 6.0++$ \\
\hline
\end{tabular}

CFA Complete Freund adjuvant. Values expressed as mean \pm SD

No significant differences among groups at baseline (Kruskal Wallis test)

$+\mathrm{p}<0.05,++\mathrm{p}<0.01,+++\mathrm{p}<0.001$ Comparisons with the positive control (Mann Whitney U test)

Treatments were given for 21 day starting at day 10 after CFA injection 
All CFA-injected positive controls exhibited the histological characteristics of joint (infiltration of mononuclear cells, pannus formation in synovial tissues, and extent of vascularity), but in none negative control, so that the score values of these findings were significantly increased in the former as compared to the negative control group (Table 3). Oral administration of D-002 $(50,200$ and $400 \mathrm{mg} / \mathrm{kg} /$ day) significantly and markedly $(91.3 \%$ in both cases) reduced the occurrence of the infiltration of mononuclear cells and pannus formation, and abolished (100\% reduction) the presence of vascularization.

While spleen samples of positive controls displayed inflammatory cells, white pulp atrophy and extramedullar hematopoyesis on the red pulp, the samples of D-002-treated rats did not show abnormalities

Table 3. First experiment: Effects of treatments on histopathological scores of rat tarsal joints

\begin{tabular}{|l|c|c|c|c|c|c|}
\hline \multirow{2}{*}{\begin{tabular}{c}
\multirow{1}{*}{$\begin{array}{c}\text { Treatment } \\
\text { groups }\end{array}$} \\
\cline { 2 - 7 }
\end{tabular}} & $\begin{array}{c}\text { Score } \\
\text { mononuclear cells }\end{array}$ & \multicolumn{2}{c|}{ Pannus formation } & \multicolumn{2}{c|}{ Vascularity } \\
\hline Sham (vehicle) & $0.0 \pm 0.0++$ & - & $0.0 \pm 0.0++$ & - & $0.0 \pm 0.0++$ & - \\
\hline $\begin{array}{l}\text { Positive control } \\
\text { (vehicle }+\mathrm{CFA})\end{array}$ & $2.90 \pm 0.35$ & - & $2.88 \pm 0.35$ & - & $2.75 \pm 0.46$ & - \\
\hline $\begin{array}{l}\text { D002 50mg/kg }+ \\
\text { CFA }\end{array}$ & $0.38 \pm 0.74+$ & 86.1 & $0.25 \pm 0.46+$ & 91.3 & $0.13 \pm 0.35+$ & 95.3 \\
\hline $\begin{array}{l}\text { D002 200mg/kg } \\
+\mathrm{CFA}\end{array}$ & $0.38 \pm 0.74+$ & 86.1 & $0.25 \pm 0.71+$ & 91.3 & $0.13 \pm 0.35+$ & 95.3 \\
\hline $\begin{array}{l}\text { D002 400mg/kg } \\
+\mathrm{CFA}\end{array}$ & $0.25 \pm 0.46+$ & 91.3 & $0.25 \pm 0.46+$ & 91.3 & $0+$ & 100 \\
\hline
\end{tabular}

CFA Complete Freund adjuvant. Values expressed as mean \pm SD

$+\mathrm{p}<0.05,++\mathrm{p}<0.01$ Comparisons with the positive control (Mann Whitney U test)

Treatments were given for 21 day starting at day 10 after CFA injection,

\section{Second experiment}

CFA-injection induced significant weight loss in the positive controls as compared to negative control group, which was significantly attenuated by both D-002 and MTX (Table 4).

Table 4. Second experiment: Effect of treatments on rat bodyweights

\begin{tabular}{|l|c|c|c|}
\hline Treatment groups & Baseline & 10 days & 28 days \\
\hline Sham (vehicle) & $327.38 \pm 17.90$ & $355.63 \pm 19.78+$ & $447.25 \pm 21.98+++$ \\
\hline Positive control (vehicle + CFA) & $324.13 \pm 29.41$ & $322.50 \pm 26.23$ & $300.38 \pm 18.01$ \\
\hline D002 $25 \mathrm{mg} / \mathrm{kg}+$ CFA & $327.88 \pm 26.49$ & $345.88 \pm 40.70$ & $400.25 \pm 57.63++$ \\
\hline D002 $100 \mathrm{mg} / \mathrm{kg}+$ CFA & $326.88 \pm 31.22$ & $353.25 \pm 34.76$ & $402.13 \pm 64.52++$ \\
\hline MTX $0.3 \mathrm{mg} / \mathrm{kg}+$ CFA & $327.38 \pm 26.48$ & $349.88 \pm 27.41$ & $396.25 \pm 88.42+$ \\
\hline \\
CFA Complete Freund adjuvant, MTX methotrexate, Values expressed as mean \pm SD \\
+ p $<0.05,++$ p $<0.01+$ p $<0.001,++$ p $<0.001$ Comparisons with the positive control (Mann Whitney U test) \\
Treatments were given for 21 day starting at day 10 after CFA injection,
\end{tabular}

CFA injection induced increase in the histological scores of infiltration of mononuclear cells, pannus formation in synovial tissues, and vascularity in the positive controls as compared to the negative control group, changes that were reversed by MTX $0.3 \mathrm{mg} / \mathrm{kg}$ (Table 5). Oral administration of D-002 significantly and markedly reduced the infiltration of mononuclear cells $(80.9 \%)$, pannus formation in synovial tissues $(81.8 \%)$ and vascularity $(90.5 \%)$, as compared to the positive control group. The effects of the highest dose of D-002 $(100 \mathrm{mg} / \mathrm{kg})$ on pannus formation and vascularity were comparable to those of MTX $0.3 \mathrm{mg} / \mathrm{kg}$, but the reduction of the infiltration of mononuclear cells with D-002 (80.9\%) was greater than with MTX (66.8\%). 
Table 5. Second experiment. Effects of treatments on histopathological scores of rat tarsal joints

\begin{tabular}{|c|c|c|c|c|c|c|}
\hline \multirow[t]{2}{*}{$\begin{array}{l}\text { Treatment } \\
\text { groups }\end{array}$} & \multicolumn{2}{|c|}{$\begin{array}{c}\text { Infiltration of } \\
\text { mononuclear cells }\end{array}$} & \multicolumn{2}{|c|}{ Pannus formation } & \multicolumn{2}{|c|}{ Vascularity } \\
\hline & Score & $\begin{array}{c}\text { Inhibition } \\
(\%)\end{array}$ & Score & $\begin{array}{c}\text { Inhibition } \\
(\%)\end{array}$ & Score & $\begin{array}{c}\text { Inhibition } \\
(\%)\end{array}$ \\
\hline Sham (vehicle) & $0.0 \pm 0.0++$ & - & $0.0 \pm 0.0++$ & - & $0.0 \pm 0.0++$ & \\
\hline $\begin{array}{l}\text { Positive control } \\
\text { (vehicle+ CFA) }\end{array}$ & $2.62 \pm 0.52$ & & $2.75 \pm 0.46$ & & $2.62 \pm 0.52$ & \\
\hline $\begin{array}{l}\text { D002 25mg/kg } \\
+ \text { CFA }\end{array}$ & $0.75 \pm 1.03+$ & -71.4 & $0.62 \pm 0.74++$ & -77.5 & $0.37 \pm 0.52++$ & -85.9 \\
\hline $\begin{array}{l}\text { D002 } 100 \mathrm{mg} / \mathrm{kg} \\
+\mathrm{CFA}\end{array}$ & $0.50 \pm 0.76++a$ & -80.9 & $0.50 \pm 0.76++$ & -81.8 & $0.25 \pm 0.46++$ & -90.5 \\
\hline $\begin{array}{l}\text { MTX } 0.3 \mathrm{mg} / \mathrm{kg} \\
+ \text { CFA }\end{array}$ & $0.87 \pm 0.99+$ & -66.8 & $0.25 \pm 0.46++$ & -90.9 & $0.25 \pm 0.46++$ & -90.5 \\
\hline
\end{tabular}

CFA Complete Freund adjuvant, MTX methotrexate, Values expressed as mean \pm SD

$+\mathrm{p}<0.01,++\mathrm{p}<0.001$ Comparisons with the positive control, a Comparisons with MTX group (Mann Whitney U test) Treatments were given for 21 day starting at day 10 after CFA injection

Inflammatory cells, white pulp atrophy, extramedullar hematopoyesis were present in spleen samples of the CFA-injected positive controls, while no disturbances were seen in the others groups (sham, D-002 and MTX-treated groups).

\section{Discussion}

In the present study, we demonstrate that oral administration of D-002 markedly reverses the histopathological findings and the decrease of exploratory activity induced by CFA, a model of RA. Our results show that D-002 (50, 200 and $400 \mathrm{mg} / \mathrm{kg} /$ day) given effectively attenuated not only the decrease of exploratory activity, but also the histopathological changes (infiltration of mononuclear cells, destruction of cartilage and bone -pannus proliferation and increase of joint vascularity) in the tarsal joint induced by the CFA injection. The effects here seen should be considered as therapeutic, not prophylactic, since treatments were given once CFA-induced arthritis was already present (day 10 after CFA injection). [33]

We conducted two experiments: a first one that evaluated the effects three doses of D-002 (50, 200 and $400 \mathrm{mg} / \mathrm{kg}$ ) given for 21 days, and a second one that assessed the effects of lower dose (25 and $100 \mathrm{mg} / \mathrm{kg}$ ) but given for a longer period (28 days), in which we introduced a group treated with MTX as reference drug, since it is recognized as the best disease-modifying anti-rheumatic drug. 8, 10 Treatments commenced always at the day 10 after CFA injection since evidences support that pathological changes typical of RA are present at such time. [28]

The behavioral experiment revealed that the injection of CFA decreased significantly the exploratory activity (functional outcome) of positive control rats as compared to the negative control group. The numbers of rears, crosses and the total exploratory activity were lowered by about $72.5 \%, 52.9 \%$ and $59 \%$, respectively, versus the values of the negative control. These results are consistent with the use of open field exploratory activity of the rats as an indirectly indicator of paw pain, and may help in assessing the potential efficacy of the treatment on the pain that accompanies the CFA-induced joint injury. [29]

All positive control rats of both experiments displayed the histopathological lesions characteristic of CFA-induced arthritis, as expected. [28] This fact, together with the ability of MTX for reversing CFA-induced lesions, confers validity to this model in our experimental conditions. The effects of D-002 on CFA-induced arthritis here seen, therefore, can be actually attributable to the treatment. In such regard, despite the difference in the dose schemes used in the two experiments the magnitude of the effects was coherent and similar, so that in both cases the 
decreases observed were actually marked $(\geq 80 \%)$ as compared to the positive control group. Perhaps the only finding to note is that in the first experiment D-002 decreased completely (100\%) the increase of joint vascularity, while in the second one this was reduced by about $90 \%$. This difference, however, falls within the logical variability between experiments, and in both cases may be considered as a remarkable effect $(\geq 90 \%)$.

The efficacy of D-002 in both experiments was grossly the same. Apparently the effects seen in the second experiment were somewhat less pronounced than in the first one, which could results from the use of lower doses. Nevertheless, the differences in the effects in both experiments were small $(\leq 10 \%)$ and thus consequence of inter-experiments variability. In addition, the effects of 50 $\mathrm{mg} / \mathrm{kg}$ given for 21 days (first experiment) were practically the same of $100 \mathrm{mg} / \mathrm{kg}$ for 28 days, suggesting a similar efficacy of both dose schemes, probably because the treatment was administered once the arthritis was already developed (day 10 after injection). [33]

CFA induced spleen lesions indicative of the impairment of this tissue induced by the adjuvant, which results from the deep induction of extramedullary hematopoiesis in the red pulp in conjunction with pyogranulomatous inflammation in the red pulp and capsule, changes usually linked with mild to marked lymphoid atrophy. $[33,34]$ The absence of spleen lesions in D-002treated animals is another evidence of its efficacy on this model, since an agent active in adjuvant disease should restore the spleen morphology to normal. [34]

Different mechanism may explain the efficacy of D-002 in this model, but obviously since it has been shown anti-inflammatory action $[16-20,23,24]$ and the ability to inhibit COX and 5LOX enzymes [21, 22] should be one of the mechanism that support the effects here seen, at least partially. Taking into account that the increase of oxidative stress has been linked with the development of RA and that some antioxidants have demonstrated efficacy in RA model, [35-38] we can hypothesize that the antioxidant effects of D-002 could have contributed to the present results, preferred hypothesis. Nevertheless, our study was not focused on the mechanisms whereby D-002 may be effective, but on elucidating whether its oral administration is able to reduce the distinctive histopathological lesions in this model of RA, as demonstrated. Then, we prefer to limit our comments to the facts here provided.

Indeed, this study presents some limitations, such as the lack of evidence of the effect of D002 on tarsal joint macroscopic inflammation, or on any relevant biochemical target, such as mieloperoxidase activity, among others. Then, although the present results are promising, they should be accepted as an introductory study of the effects of D-002 on this subject, since extensive further investigation on this matter is required.

\section{Conclusions}

This is the first report demonstrating the efficacy of oral treatment with D-002 in a rat model of antigen-induced arthritis. The effects here described should be considered as therapeutic, not prophylactic, since treatments were given once CFA-induced arthritis was already present (day 10 after CFA injection). In the first experiment D-002 (50, 200 and $400 \mathrm{mg} / \mathrm{kg} /$ day) was administered for 21 days and in the second experiment during 28 days and both effectively attenuated not only the decrease of body weight and exploratory activity used as an indirectly marker of paw pain, but also the histopathological changes (infiltration of mononuclear cells, destruction of cartilage and bone -pannus proliferation and increase of joint vascularity) in the tarsal joint induced by the CFA injection. These changes were significantly and markedly ameliorated by D-002 as compared to the positive control. MTX as reference drug also reversed CFA-induced changes. The reduction of the infiltration of mononuclear cell with D-002 $400 \mathrm{mg} / \mathrm{kg}$ was greater $(80.9 \%)$ than with MTX $(66.8 \%)$, but effects on other variables were similar. Inflammatory cells, white pulp atrophy, extramedullar hematopoyesis were present in spleen samples of the CFA-injected positive controls, while no disturbances were seen in the other groups (sham, D-002 and MTX-treated groups). 


\section{References}

[1]. M. Cutolo, G.D. Kitas, PL. van Riel, Burden of disease in treated rheumatoid arthritis patients: going beyond the joint, Semin. Arthritis Rheum. 43 (2014) 479-488.

[2]. E. Salgado, J.R. Maneiro, New therapies for rheumatoid arthritis, Med. Clin. (Barc). 13 (2014) 841-845.

[3]. H. Radner, J.S. Smolen, D. Aletaha, Impact of comorbidity on physical function in patients with rheumatoid arthritis, Ann. Rheu. Dis. 69 (2010) 536-541.

[4]. E. Lindqvist et al., Ten year outcome in a cohort of patients with early rheumatoid arthritis: health status, disease process, and damage, Ann. Rheu. Dis. 61(2002) 1055-1059.

[5]. H. Radner, J.S. Smolen, D. Aletaha, Remission in rheumatoid arthritis: benefit over low disease activity in patient reported outcomes and costs, Arthritis Res. Ther. 16 (2014) R56.

[6]. CL. Overman et al., Change of psychological distress and physical disability in patients with rheumatoid arthritis over the last two decades, Arthritis Care Res. (Hoboken). 66(5) (2014) 671-678.

[7]. H. To, Rheumatoid arthritis, Nihon Rinsh. 71 (2013) 2141-2145.

[8]. D. Palmer, Y. El Mledany, Treat-to-target: a tailored treatment approach to rheumatoid arthritis, Br. J. Nurs. 22 (2013) 312-318.

[9]. O. Ethgen et al., What do we know about the safety of corticosteroids in rheumatoid arthritis? Curr. Med. Res. Opin. 29 (2013) 1147-1160.

[10]. P. Cipriani et al., Methotrexate in rheumatoid arthritis: optimizing therapy among different formulations. Current and emerging paradigms, Clin. Ther. 36 (2014) 427-435.

[11]. LJ. Crofford, Use of NSAIDs in treating patients with arthritis, Arthritis Res. Ther. 15(3) (2013) S2.

[12]. EL. Massó González et al., Variability among non-steroidal antiinflammatory drugs in risk of upper gastrointestinal bleeding, Arthritis and Rheumatism. 15 (2010) 1592-1601.

[13]. M. Hegen et al., Utility of animal models for identification of potential therapeutics for rheumatoid arthritis, Ann. Rheum. Dis. 67 (2008) 1505-1515.

[14]. DL. Asquith et al., Animal models of rheumatoid arthritis, Eur. J. Immunol. 39 (2009) 20402044.

[15]. A. Beresniak et al., Interest of modelling in rheumatoid arthritis, Clin. Exp. Rheumatol. 4 Suppl. 73 (2012) S96.

[16]. D. Carbajal et al., Anti-inflammatory activity of D-002: an active product isolated from beeswax, Prostaglandins Leukot Essent Fatty Acids. 59 (1998) 235-238.

[17]. D. Carbajal et al., Effect of D-002 on models of acute inflammation, IJPSRR 21 (2013) 62-67.

[18]. Y. Ravelo et al., Effects of single oral and topical administration of D-002 (beeswax alcohols) on xylene induced ear edema in mice, Latin American Journal of Pharmacy. 29 (2010) 14511454.

[19]. Y. Ravelo et al., Evaluation of antiinflammatory and antinociceptive effects of D-002 (beeswax alcohols), J. Nat. Med. 65 (2010) 330-335.

[20]. Y. Perez et al., Effect of D-002 on 5-lypooxygenase activity in vitro, Rev. Cubana Farm. 46 (2012) 259-266.

[21]. Y. Pérez et al., Inhibition of ciclooxygenase and 5-lipooxygenase enzymes by D-002 (beeswax alcohols), Top Current Nutraceutical Res. 12 (2014) 13-18.

[22]. S. Mendoza et al., Effects of D-002 on formaldehyde-induced osteoarthritis in rats. IOSR J. Pharm. 3 (2013) 9-12.

[23]. S Mendoza et al., Ameliorating effects of D-002, a mixture of beeswax alcohols, on monosodium iodoacetate-induced osteoarthritis in rats, Int. J. Pharm. Sci. Rev. Res. 19 (2013) $10-15$.

[24]. I. Rodríguez et al., Effects of D-002, a mixture of beeswax alcohols, on osteoarthritis symptoms: a randomized placebo-controlled study, IOSR J. Pharm. 2 (2012) 1-9.

[25]. R. Puente et al., Evaluation of the effect of D-002, a mixture of beeswax alcohols, on arthritic symptoms, The Korean journal of internal medicine. 29 (2014) 191-202. 
[26]. K. Bauerova et al., Utilization of adjuvant arthritis model for evaluation of new approaches in rheumatoid arthritis therapy focused on regulation of immune processes and oxidative stress, Interdisc. Toxicol. 4 (2011) 33-39.

[27]. A. Al-Abda et al., Nimesulide improves the disease modifying anti-rheumatic profile of methotrexate in mice with collagen-induced arthritis, European J. Pharmacol. 644 (2010) 245250.

[28]. Y. Haruna et al., Fluvastatin reverses endothelial dysfunction and increased vascular oxidative stress in rat adjuvant-induced arthritis. Arthritis \& Rheumatism. 56 (2007) 1827-1835.

[29]. U. Heilborn et al., Spontaneous nociceptive behaviour in female mice with Freund's complete adjuvant- and carrageenan-induced monoarthritis, Brain Res. 27 (2007) 143-149.

[30]. D. Carbajal et al., Efecto del D-004 sobre patrones conductuales en roedores, Rev. CENIC Ciencias Biológicas. 38 (2007) 12-17.

[31]. L. Mosekilde, C. Danielsen, U.B. Knudsen, The effect of aging and ovariectomy on the vertebral bone mass and biomechanical properties of mature rats, Bone. 14 (1993) 1-6.

[32]. A. Omoto et al., Copper chelation with tetrathiomolybdate suppresses adjuvant-induced arthritis and inflammation-associated cachexia in rats, Arthritis Res. Ther. 7 (2005) R1174R1182.

[33]. AM. Bendele, Animal models of rheumatoid arthritis, J. Musculoskel Neuron Interact. 1 (2001) 377-385.

[34]. R. Jaimik et al., Ameliorative effect of Bambusa arundinacea against adjuvant arthritis-with special reference to bone erosion and tropical splenomegaly, J. of Drug Delivery and Therap. 2 (2012) 141-145.

[35]. Y. Ozkan et al., Oxidative status in rheumatoid arthritis, Clin. Rheumatol. 26(1) (2007) 6468.

[36]. A. Nakajima et al., Identification of clinical parameters associated with serum oxidative stress in patients with rheumatoid arthritis, Mod. Rheumatol. 24 (2014) 926-930.

[37]. M. Veselinovic et al., Oxidative stress in rheumatoid arthritis patients: relationship to diseases activity, Mol. Cell Biochem. 391 (2014) 225-232.

[38]. RM. El-Sayed, YM. Moustafa, MF. El-Azab, Evening primrose oil and celecoxib inhibited pathological angiogenesis, inflammation, and oxidative stress in adjuvant-induced arthritis: novel role of angiopoietin-1, Inflammopharmacology. 5 (2014) 305-317. 